Geistige Eigentumsrechte, was steht für die Entwicklungsländer auf dem spiel?

\title{
TRIPS-Abkommen : eine Herausforderung für die Entwicklungsländer
}

Nadine Keim

\section{(2) OpenEdition}

\section{Journals}

Electronic version

URL: http://journals.openedition.org/sjep/684

DOI: $10.4000 /$ sjep.684

ISSN: 1663-9677

\section{Publisher}

Institut de hautes études internationales et du développement

\section{Printed version}

Date of publication: 1 avril 1998

Number of pages: $65-73$

ISSN: $1660-5926$

Electronic reference

Nadine Keim, «TRIPS-Abkommen : eine Herausforderung für die Entwicklungsländer »,

Schweizerisches Jahrbuch für Entwicklungspolitik [Online], 17 | 1998, Online erschienen am: 01 August

2012, abgerufen am 08 September 2020. URL : http://journals.openedition.org/sjep/684 ; DOI :

https://doi.org/10.4000/sjep.684 


\title{
TRIPS-ABKOMMEN: EINE HERAUSFORDERUNG FÜR DIE ENTWICKLUNGSLÄNDER
}

\author{
NADINE KEIM COMTE*
}

S EIT dem Bestehen der Welthandelsorganisation (WTO) haben die Länder des Südens keine Möglichkeit mehr, von anderen erfundene Techniken nachzuahmen. Abgesehen von wenigen Ausnahmen sieht das TRIPsAbkommen vor, dass technologische Erfindungen patentrechtlich geschützt werden müssen. Ausserdem verpflichtet das Abkommen die Unterzeichnerstaaten dazu, Mikroorganismen zu patentieren sowie ein anderweitiges Schutzsystem für Pflanzensorten auszuarbeiten. Die einzige Art geistigen Eigentums, die künftig keinerlei Schutz geniessen wird, sind die traditionellen Kenntnisse der Länder des Südens. Die Folgen sind absehbar: Das allgemein anerkannte und geltende Patentsystem führt dazu, dass selbst eventuelle Kompensationszahlungen, die ein Industrieland aus eigenem Antrieb zu leisten bereit wäre, verunmöglicht werden. Für die Entwicklungsländer bedeutet dies administrative Auflagen und zusätzliche Kosten, und nicht zuletzt stellt es aus gesetzgeberischer und ethischer Sicht eine Herausforderung dar.

Mit einem Patent kann ein Erfinder ein ausschliessliches kommerzielles Recht in bezug auf seine Erfindung geltend machen. Das Recht ist zeitlich begrenzt und wird im Austausch gegen die sofortige Veröffentlichung der Geheimnisse der Erfindung gewährt. Dieses System stellt einen Kompromiss zwischen gerechtfertigten Kompensationsleistungen an den Erfinder und dem Allgemeinwohl dar, der Weiterentwicklungen und neue Erfindungen erlauben soll. Das Patent, das ursprünglich als Mittel zur Förderung von Forschung und Industrialisierung gedacht war, entwickelte sich zunehmend zu einem Monopol- und Kontrollinstrument zugunsten der technologisch fortgeschrittenen Industrien des Nordens. So werden weltweit rund $90 \%$ aller Patente in den Ländern des Nordens angemeldet, und mehr als die Hälfte der in den südlichen Ländern hinterlegten Patente sind im Besitz von Ausländern.

Ein zu strenger Schutz des geistigen Eigentums kann die wirtschaftliche Entwicklung eines Landes bremsen. Sobald jedoch ein Staat die Schwelle zum Industrieland überschritten hat, wird er in der Regel zu einem eifrigen Verfechter des Patents. Mit den Fortschritten in der Bio- und Gentechnologie haben sich die Entwicklungskosten für neue Erfindungen laufend verteuert, wohingegen sich das Kopieren dieser Erfindungen nach wie vor relativ einfach, rasch und kostengünstig bewerkstelligen lässt. Angesichts der Intensivierung der Handelsbeziehungen und insbesondere der wachsenden Furcht des Nordens vor dem Verlust seiner marktbeherrschenden Stellung und seiner Wettbewerbsfähigkeit dürfte der Schutz des geistigen Eigentums auch weiterhin an Bedeutung gewinnen.

* Entwicklungspolitische Koordination, Arbeitsgemeinschaft Swissaid/Fastenopfer/Brot für alle/Helvetas/Caritas, Lausanne. 


\section{DER FALL SCHWEIZ: ENTWICKLUNG DANK KOPIEN AUSLÄNDISCHER ERFINDUNGEN}

Im vergangenen Jahrhundert haben sich zahlreiche Schweizer Industrielle gegen den Schutz des geistigen Eigentums gewehrt. Das erste einschlägige Gesetz trat denn auch erst 1988 in Kraft. Während Jahrzehnten galt unser Land weltweit als Nation von Piraten: Die chemische Industrie zögerte nie, die Produkte und Verfahren ausländischer Konkurrenten zu imitieren. So beklagte die deutsche Regierung im Jahre 1904 die «helvetischen Plünderungen» deutscher Erfindungen wie z.B. Aspirin oder Heroin. Die Unternehmen konnten so auf Forschungs- und Entwicklungskosten verzichten und gleichzeitig ihre Kopien billiger als die Originale verkaufen. Hätte die pharmazeutische Industrie diese Möglichkeit nicht genutzt, so hätte sich ihre Entwicklung zu einem wettbewerbsfähigen Industriezweig weit schwerer und teurer gestaltet. Erst seit $1978^{1}$ kennt die Schweiz den Schutz von Medikamenten als Fertigprodukte.

Noch nie hat die Entwicklungsgeschichte eines Landes den Beweis erbringen können, dass der patentrechtliche Schutz von Erfindungen eine Schlüsselrolle in der wirtschaftlichen Entwicklung spielt. Im Gegenteil: Im Falle Japans, Koreas oder Taiwans konnten wirtschaftliche Erfolge erzielt werden, indem ausländische Erfindungen imitiert wurden. Diese Möglichkeit bestand damals, weil kein modernes Patentsystem existierte. Das Patent steht in einem krassen Gegensatz zu den heutzutage allgemein anerkannten Prinzipien der Konkurrenz und der Marktwirtschaft. Die liberalen Denker der Anfänge waren sich dieses Widerspruchs bewusst, und der Erfolg der Anhänger des Patents im vergangenen Jahrhunderts bedeutete den Sieg des Protektionismus. Man sprach damals vom natürlichen Recht zur Nachahmung.

\section{DEM SÜDEN BLEIBT KEINE WAHL}

Ein Erzeugnis, das in einem bestimmten Land patentiert wurde, ist in der Regel in den Nachbarstaaten nicht geschützt. Dies ist ein Grund dafür, weshalb die Staaten eine engere Zusammenarbeit auf diesem Gebiet anstreben. Der Grundstein für diese Kooperation wurde 1883 mit dem Abschluss der Pariser Verbandsübereinkunft zum Schutz des gewerblichen Eigentums gelegt. Diese Übereinkunft sowie die nachfolgenden Abkommen, die heute von der WIPO (Weltorganisation für geistiges Eigentum) verwaltet werden, genügen jedoch nach Ansicht der Industrieländer den heutigen Anforderungen nicht mehr. Das TRIPs-Abkommen ( Abkommen über handelsbezogene Aspekte der Rechte an geistigem Eigentum») vereint die ersten zwingenden und weltweit harmonisierten Normen auf diesem Gebiet (Art. 27.1 und 33) und stellt eine der wichtigsten Säulen der WTO (Welthandelsorganisation) dar, die seit dem 1. Januar 1995 besteht und über 130 Mitgliedsstaaten zählt.

Das TRIPs-Abkommen verpflichtet die Mitgliedsstaaten, eine technologische Erfindung während mindestens 20 Jahren patentrechtlich zu schützen. Die Erfindung muss eine Neuheit darstellen, von einer erfinderischen bzw. nicht naheliegenden Tätigkeit herrühren und gewerblich anwendbar oder nützlich sein. Im allgemeinen wird die Anwendung des Abkommens vom 1. Januar 2000

1. Verordnung über die Erfindungspatente (Patentverordnung) vom 19. Oktober 1977. 
an alle zwei Jahre durch die WTO überprüft (Art. 71). Als Folge davon besitzen die Länder des Südens nicht mehr die Möglichkeit, von anderen erfundene Techniken nachzuahmen oder anzupassen, wie es für die reichen Länder zum Zeitpunkt ihrer Industrialisierung noch der Fall war. Künftig werden arme Länder, die Technologien importieren, sogar Lizenzgebühren für gewisse Nahrungsmittel oder als unabdingbar eingestufte Medikamente bezahlen müssen.

\section{FLEXIBILITÄT JA, ABER VOR ALLEM KOSTEN}

Immerhin sind für die Entwicklungsländer Übergangszeiten vorgesehen worden. Nach Inkrafttreten der WTO haben sie fünf Jahre, d.h. bis zum 1. Januar 2000, um minimale patentrechtliche Normen einzuführen. Darüber hinaus ist für pharmazeutische und agrochemische Erfindungen eine zusätzliche Frist von nochmals fünf Jahren vorgesehen (Art. 65), wobei jedoch die ausschliesslichen Vermarktungsrechte von Anfang an gewährt werden müssen (Art. 70). Für die am wenigsten entwickelten Länder wurde das Datum des Inkrafttretens auf den 1. Januar 2006 gelegt, Fristverlängerungen über dieses Datum hinaus sind auf Ersuchen hin möglich (Art. 66).

Aber auch neben diesen Übergangsperioden sind im TRIPs-Abkommen zumindest auf dem Papier - einige Ausnahmeregelungen vorgesehen, die es den Ländern des Südens erlauben sollten, ihre eigenen Entwicklungsinteressen zu verfolgen. Erstens steht es den Unterzeichnerstaaten offen, «Massnahmen zur Förderung des öffentlichen Interesses in den für ihre sozioökonomische und technologische Entwicklung entscheidend wichtigen Sektoren»zu ergreifen (Art. 8). Zweitens ist die Gewährung des patentrechtlichen Schutzes nicht obligatorisch, wenn er dem «Schutz der öffentlichen Ordnung oder der guten Sitten einschliesslich des Schutzes des Lebens oder der Gesundheit von Menschen, Tieren oder Pflanzen» zuwiderlaufen oder «eine schwere Schädigung der Umwelt» bedeuten würde. (Art. 27.2). Das Recht, patentierte Erfindungen kostenlos zu Forschungszwecken nichtkommerzieller Natur zu verwenden, ist ebenfalls garantiert (Art. 30).

In gewissen Fällen haben die Länder auch die Möglichkeit, ein Patent ohne Erlaubnis des Rechtsinhabers zu verwenden (Art. 31). Es handelt sich dabei um die sogenannten «Zwangslizenzen», deren Verwendung im TRIPs-Abkommen zwar nicht untersagt, aber doch an strenge Bedingungen gekoppelt wird. So könnte beispielsweise ein Land zugunsten des öffentlichen Interesses oder der öffentlichen Gesundheit, in einer nationalen Notstandssituation, zum öffentlichen nichtgewerblichen Gebrauch oder zur Bekämpfung des unlauteren Wettbewerbs eine Zwangslizenz gewähren. Im selben Zusammenhang besteht auch die Möglichkeit, nationale Massnahmen zur Bekämpfung wettbewerbswidriger Praktiken seitens der Privatwirtschaft zu ergreifen (Art. 40). Die Zukunft wird zeigen, ob die armen Länder diese Ausnahmebestimmungen in ihrem eigenen Interesse nutzen können, und vor allem, ob es ihnen gelingen wird, bei Streitigkeiten die Ergreifung solcher Massnahmen im Rahmen der WTO zu rechtfertigen. Jedenfalls dürfte klar sein, dass nur jene Länder ihre Rechte durchsetzen werden, die sich auf eine schlagkräftige und spezialisierte Verwaltung stützen können. 
Vor allem aber bedeuten die WTO-Bestimmungen für die Entwicklungsländer zusätzliche administrative Auflagen. Allein für Bangladesh werden die zusätzlichen Kosten für die Errichtung patentrechtlicher Mindestnormen auf über eine Million Dollar pro Jahr geschätzt. Darunter fallen insbesondere die Ausarbeitung gesetzlicher Bestimmungen, die Einrichtung einer spezialisierten Behörde, die Ausbildung von Sachverständigen sowie die Verteidigung der Interessen des Landes innerhalb der WTO. Das TRIPs-Abkommen stellt demnach für die Länder des Südens eine grosse gesetzgeberische Herausforderung dar. Für sie hängt sehr viel davon ab, ob die von den westlichen Ländern gemachten Zusagen hinsichtlich der technischen Zusammenarbeit in die Tat umgesetzt werden, insbesondere im Zusammenhang mit der Ausarbeitung von Gesetzen, der Unterbindung von Missbräuchen, der Unterstützung der Verwaltung und der Ausbildung des Personals (Art. 67), aber auch im Hinblick auf den Technologietransfer (Art. 66).

Bis heute konnten die Entwicklungsländer auf dem Gebiet des geistigen Eigentums in den Bereichen Beratung, Ausbildung und Bereitstellung von Material auf die unterstützende technische Zusammenarbeit der WIPO zählen. Dies genügt allerdings nicht mehr. In Zukunft müssen die Industrieländer sowohl auf bilateraler Ebene als auch durch kompetente multilaterale Organe zusätzliche Anstrengungen unternehmen, damit der Süden seine Gesetzgebung in diesem Bereich so gestalten kann, dass sie seinen eigenen Entwicklungsbedürfnissen gerecht wird. Die Schweiz als eines der Länder mit der höchsten Patentdichte ist dabei ganz besonders gefordert. Allerdings hat sie bei den Verhandlungen über das TRIPs-Abkommen auf der Seite der USA und der Europäischen Union die Position der meisten Entwicklungsländer bekämpft.

\section{DAS TOR ZUM PATENT AUF LEBEN}

Eines der umstrittensten Themen bei den Verhandlungen über das TRIPsAbkommen war die Ausdehnung der Patentierbarkeit auf lebende Organismen. Schliesslich konnten sich die Mitgliedsstaaten der WTO auf einen Kompromiss einigen, der ein treffendes Beispiel für die Rechtsauslegung in den westlichen Ländern darstellt: Mikroorganismen sowie mikrobiologische und technische Verfahren müssen patentiert werden (Art. 27.3 b). So sind die Mitgliedsländer beispielsweise verpflichtet, ein genetisch verändertes Bakterium patentrechtlich zu schützen. Der Schutz des Lebens durch ein Patent wird demnach im TRIPsAbkommen ausdrücklich festgehalten, selbst wenn der Schutz von Pflanzen, Tieren und natürlichen Prozessen nicht verbindlich ist. Immerhin wurde beschlossen, «den Schutz von Pflanzensorten entweder durch Patente oder durch ein wirksames System sui generis oder durch eine Verbindung beider» vorzusehen (Art. $27.3 \mathrm{~b}$ ).

In anderen Worten: Die Staaten sind nicht zum Schutz von Pflanzenzüchtungen durch Patente gezwungen, aber wenn sie sich gegen das Patent entscheiden, sind sie verpflichtet, diesen Schutz durch ein anderes Mittel ihrer Wahl zu gewähren. Die Verhandlungspartner hatten dabei an das internationale Übereinkommen zum Schutz von Pflanzenzüchtungen (UPOV) gedacht (siehe weiter unten), aber nichts spricht dagegen, dass es sich nicht auch um ein eigenständiges System handeln könnte. Der Haken an der Sache ist, dass die Möglichkeit 
zur Errichtung eines solchen Systems, welches den nationalen und entwicklungspolitischen Interessen des betreffenden Landes gerecht wird, ausgeschaltet werden könnte, bevor sie überhaupt wahrgenommen werden kann, denn erstens ist die WTO verpflichtet, die diesbezüglichen Bestimmungen in Art. $27.3 \mathrm{~b}$ bis zum 1. Januar zu überprüfen, und zweitens muss gemäss denselben Bestimmung der Schutz von Pflanzenzüchtungen durch ein derartiges «wirksames System sui generis» frühestens ab dem 1. Januar 2000 gewährleistet sein.

Zur Zeit bestehen neben dem Patentrecht und dem UPOV-System keine weiteren Möglichkeiten zum Schutz von Pflanzenzüchtungen, und die Tatsache, dass die Begriffe «sui generis» und, mehr noch, "wirksam» alles andere als klar definiert sind, macht die Sache für die Länder des Südens nicht einfacher. Vieles bleibt noch zu tun, um den westlichen Ländern zu beweisen, dass die Schaffung anderer Systeme auch tatsächlich möglich ist. Indien, Thailand, die Philippinen sowie die Mitgliedsstaaten des Andenpaktes haben den Sprung ins kalte Wasser gewagt und mit der Ausarbeitung autonomer Gesetze begonnen. Die USA verfolgen diese Aktivitäten mit Misstrauen: Im Falle Thailands beispielsweise schrecken amerikanische Diplomaten nicht davor zurück, bereits jetzt Kritik zu äussern.

Daraus wird klar, dass sich der patentrechtliche Schutz, der ursprünglich für Erfindungen aus dem Bereich der Physik geschaffen wurde, langsam aber sicher auf lebende Organismen ausdehnt. Pflanzen und Tiere werden damit zunehmend zum geistigen Eigentum im Dienste privater Interessen. Die weltweit einzige Art von geistigem Eigentum, die in keinerlei Hinsicht geschützt ist, dürfte künftig das traditionelle Wissen der Völker des Südens sein. Damit würde eine grundlegende Ungerechtigkeit geschaffen, die die Entwicklungsländer mit zwei zusätzlichen Problemstellungen konfrontiert.

\section{LANDWIRTEPRIVILEG ZUNEHMEND MISSACHTET}

Bis heute deckt die arme bäuerliche Bevölkerung in den südlichen Ländern gegen $80 \%$ ihres Bedarfs an Saatgut durch traditionelle Tauschgeschäfte. Auf diese Weise können die Bauern ihre Kosten und ihre Abhängigkeit von auswärtigen Lieferanten gering halten. Diese Vorgehensweise wird jedoch durch den Schutz des geistigen Eigentums in Frage gestellt: Was seit jeher ein unveräusserliches Recht war, ist in den 80er Jahren ein vom politischen Klima abhängiges «Privileg» geworden. Wird dieses abgeschafft, würde der Bauer, der eine modifizierte Maissorte anbaut und einen Teil der Ernte als Saatgut für das kommende Jahr zurückbehalten möchte, jedesmal eine Gebühr entrichten müssen.

Seit 1961 besteht das «Internationale Übereinkommen zum Schutz von Pflanzenzüchtungen» (UPOV). Im Unterschied zum Patent sollte dieses Schutzinstrument ursprünglich den ungehinderten und gebührenfreien Zugang zu verbesserten Erfindungen zu nichtkommerziellen Zwecken garantieren. Zum einen stand es den Bauern frei, Saatgut für eine geschützte Pflanzensorte zu tauschen oder für die nächste Aussaat wiederzuverwenden («Landwirteprivileg»), und zum anderen war es möglich, eine geschützte Sorte als Ausgangsmaterial für die Züchtung einer neuen Sorte und deren gewerbsmässige Verbreitung zu verwenden («Einverständnis des Züchters»). Bei der letzten Revision des UPOV- 
Übereinkommens im Jahre 1991 wurden diese Vorteile jedoch stark eingeschränkt, und die Entscheidungen über das «Landwirteprivileg» wurden sogar vollkommen von der jeweiligen nationalen Gesetzgebung abhängig gemacht. Ein weiteres schwerwiegendes Problem ist, dass die traditionell als Saatgut verwendeten Pflanzensorten in der neuen Fassung des UPOV-Übereinkommens nicht geschützt werden, mit der Begründung, dass diese weder neu noch stabil seien (d.h. sie können sich durch die Reproduktion über mehrere Generationen hinweg modifizieren). Tatsache ist jedoch, dass diese Pflanzensorten in zahlreichen Fällen genau den Bedürfnissen der jeweiligen Bevölkerung entsprechen und diesen auch in Zukunft und vielleicht besser als Sorten aus der modernen Forschung gerecht würden.

Nur acht Länder des Südens (von 34) sind dem UPOV-Übereinkommen von 1978 beigetreten, und die letzte Version von 1991 ist noch nicht in Kraft. Das UPOV-Generalsekretariat versucht zur Zeit, mit den Ländern des Südens in Kontakt zu treten und sie für die neueste Version dieses Übereinkommens zu gewinnen. Diese ist jedoch nicht unbedingt die ideale Lösung für jene Länder, die die Anerkennung des «Landwirteprivilegs» und der traditionellen Pflanzensorten anstreben. Sollte sich die Frage nach einem Beitritt zu diesem Übereinkommen stellen, so wäre aus dieser Sicht die Version von 1978 auf jeden Fall vorzuziehen (was möglich ist, solange die Fassung von 1991 noch nicht in Kraft getreten ist).

\section{RECHTE DER BAUERN EXISTIEREN NUR IN DER THEORIE}

Die Bestände an wilden Pflanzen und Tieren in den Ländern des Südens können mittlerweile als die neuen Ressourcen der Gegenwart bezeichnet werden. Die «Erfindungen» der autochthonen Völker basieren zwar auf in der freien Natur vorkommendem Material, sind aber in dieser Form dort nicht vorhanden, sondern stellen das Produkt menschlichen Wissens dar. Damit eine Pflanze als Heilmittel verwendet werden kann, müssen die Eigenschaften der betreffenden Sorte, ihre Standorte, die verwertbaren Teile, die Präparierung, die nötigen Zutaten, ihre Posologie usw. genauestens bekannt sein.

Die derzeit gültigen Bestimmungen über das geistige Eigentum erlauben es den multinationalen Unternehmen, sich die Erfindungen der autochthonen Völker ohne die geringste Gegenleistung anzueignen. Die Länder des Südens stellen den grössten Teil der biologischen Vielfalt unseres Planeten, aber es sind die multinationalen Unternehmen der nördlichen Industrieländer, die sich daran bereichern, ohne einen Rappen dafür zu bezahlen. So können sie die modifizierten Sorten patentieren lassen und anschliessend zu beachtlichen Preisen verkaufen, nicht selten sogar in das Herkunftsland dieser Pflanzen selbst. Laut Schätzungen der Vereinten Nationen beläuft sich der durch unbezahlte Lizenzgebühren verursachte Einkommensverlust der südlichen Länder auf mehr als fünf Milliarden Dollar pro Jahr.

1983 haben die Mitgliedsstaaten der FAO das «Engagement international sur les ressources phytogénétiques» ins Leben gerufen. Es handelt sich dabei um ein nicht zwingendes Übereinkommen zwischen mehr als 100 Ländern mit dem Ziel, den Schutz und die Nutzung von Pflanzen zu regeln. Der Grundgedanke 
dieses Abkommens wird in Art. 1 dargelegt und lautet: Genetische Ressourcen pflanzlicher Herkunft sind Teil des Erbes der Menschheit und müssen demnach ohne Einschränkung genutzt werden können. 1981 wurde das Übereinkommen um einen Anhang erweitert, welcher die «Rechte der Bauern» anerkennt, d.h. die in der Vergangenheit, gegenwärtig oder zukünftig geleisteten Anstrengungen zur Bewahrung, Verbesserung und zur Verfügbarkeit genetischer Ressourcen pflanzlicher Herkunft (Anhang II).

\section{RECHT GEGEN VERSPRECHEN}

Seither wurden kaum nennenswerte Anstrengungen unternommen, um die «Rechte der Bauern» zu konkretisieren. Eine der grössten Schwierigkeiten besteht darin, den Beitrag oder das Wissen der einheimischen Völker "gerecht» zu beziffern; ausserdem ist noch keineswegs klar, wem und in welcher Form diese Kompensationsleistungen auszuzahlen sind. Nicht zuletzt bestehen in den westlichen Staaten und in den traditionellen Gesellschaftsformen teilweise grundlegend verschiedene Auffassungen von Eigentum: Die Länder des Südens kennen häufig kein individuelles, sonder nur kollektives Eigentum. Der internationale Fonds der FAO für Ausgleichszahlungen für die Rechte der Bauern ist ebenfalls noch nicht tätig geworden.

Das «Übereinkommen über die biologische Vielfalt» und die «Agenda 21 », welche 1992 anlässlich der Umweltkonferenz in Rio unterzeichnet wurden, haben nichts zur Klärung dieser Situation beitragen können. Das erstere, welches zwingenden Charakter besitzt, anerkennt auf dem Papier die nationale Souveränität über die biologischen Ressourcen (Art. 15), bestätigt die Rolle und die Rechte der einheimischen Völker hinsichtlich der Nutzung von pflanzlichen Ressourcen (Art. 8) und sieht eine gerechte Aufteilung der Gewinne in Form von Zugang zu neuen Technologien und vorrangiger Teilnahme an der Forschung für den Süden vor (Art. 16 und 19). Die Anwendung dieser Prinzipien, insbesondere im Zusammenhang mit dem geistigen Eigentum, wird im Übereinkommen jedoch nicht klar geregelt. Die Schweiz, die Vereinigten Staaten sowie die Europäische Union haben das Übereinkommen ebenfalls unterzeichnet, unter dem Vorbehalt jedoch, dass beim Technologietransfer die Patente respektiert werden.

Nach wie vor steht also das anerkannte und geltende Patentsystem den eventuellen, vom guten Willen der reichen Länder abhängigen Kompensationsversprechungen gegenüber. Und nach wie vor existieren weitreichende Divergenzen zwischen den Ländern, die ein einfach zu handhabendes Kompensationssystem bevorzugen und jenen, die eine regelrechte Gesetzgebung über die «Rechte der Bauern » anstreben. Danach sollten die Bauern

- als Produzenten das Recht besitzen, geschütztes Saatgut für die nächste Aussaat zu verwenden bzw. auszutauschen,

$\checkmark$ als Eigentümer von Ressourcen das Recht besitzen, die Pflanzen und das damit verbundene Wissen zu kontrollieren und Zugang zu Forschungsergebnissen zu erhalten, und

- als Akteure bei der Bewahrung und Verbesserung der biologischen Vielfalt das Recht besitzen, für ihre Anstrengungen belohnt $\mathrm{zu}$ werden sowie einen gerechten Anteil an den Gewinnen zu erhalten. 


\section{WELCHE RECHTE FÜR KOLLEKTIVE INNOVATIONEN?}

Das Patent, vor mehr als einem Jahrhundert von den westlichen Nationen zum Schutz ihrer technischen Erfindungen geschaffen, kann den Beitrag, den die einheimischen Völker zum Wissen der Menschheit geleistet haben, nicht schützen. Die «Privilegien» und «Rechte der Bauern» stellen insofern eine rechtliche Neuerung dar, als sie keine neuen Erfindungen, sondern eine bewahrende Tätigkeit und eine Tradition begünstigen. Ein solches Recht, welches nach westlicher Rechtsauffassung im Widerspruch zum Begriff des «geistigen Eigentums» steht, muss als Ergänzung zu den bereits bestehenden Normen betrachtet werden. In der Folge stehen die Länder des Südens vor einer doppelten Herausforderung: Sie müssen ein System zum Schutz des geistigen Eigentums ausarbeiten, welches sich von bestehenden Regelungen unterscheidet, und gleichzeitig müssen die kollektiven Rechte in dieses System eingebaut werden. Ein solches Regelwerk könnte sich beispielsweise an folgenden Vorbildern orientieren :

- Die "dispositions types sur le folklore» der UNESCO/WIPO aus dem Jahr 1985 enthalten drei neuartige Elemente, welche für den Schutz von Pflanzen besonders geeignet wären. Das Kollektiv kann (anstelle des Einzelnen) als Erfinder registriert werden. Kollektive Erfindungen können evolutiven Charakter besitzen (d.h. ihre Entwicklung ist nicht abgeschlossen) und trotzdem geschützt werden. Solange es seine erfinderische Tätigkeit fortsetzt, besitzt das Kollektiv die ausschliessliche Kontrollgewalt über sein Erbe.

- Das Projekt zur «Deklaration der Vereinten Nationen zu den Rechten der indigenen Völker» aus dem Jahr 1994 ruft dazu auf, das Wissen, die Kultur und die überlieferten Erfahrungen der indigenen Völker anzuerkennen. Diese erhalten das Recht, Ressourcen zu besitzen (Art. 26) und ihre Wissenschaft einschliesslich der genetischen Ressourcen, des Saatguts, der Medizin und des überlieferten Wissens zu schützen (Art. 29).

- Die «Konvention 169 über indigene Völker und Stämme» der ILO aus dem Jahr 1989, welche den einheimischen Völkern das Eigentumsrecht über das traditionell von ihnen bewirtschaftete Land zuerkennt (Art. 14). Die Konvention garantiert ihre Rechte an den natürlichen Ressourcen dieses Landes, einschliesslich das Recht, an der Nutzung, Verwaltung und Bewahrung dieser Ressourcen teilzunehmen (Art. 15).

\section{NEUE HERAUSFORDERUNGEN}

Die Arbeitsgruppe Swissaid/Fastenopfer/Brot für alle/Helvetas/Caritas hat in einem kürzlich erschienenen Dokument ihre Überlegungen zum Thema Patente aus entwicklungspolitischer Sicht dargelegt und insbesondere folgende Forderungen gestellt:

- Die Länder des Südens müssen die Übergangszeit nutzen, die ihnen laut TRIPs-Abkommen zusteht, um eigene patentrechtliche Normen auszuarbeiten.

\ Die Länder des Südens müssen die Flexibilität des TRIPs-Abkommens ausschöpfen, um ihre Interessen so weit als möglich geltend zu machen: Ausnahmen zum Schutz der öffentlichen Ordnung, Zwangslizenzen und Massnahmen gegen wettbewerbswidrige Praktiken. 
口 Die Länder des Südens brauchen Zusagen zur technischen und finanziellen Zusammenarbeit seitens des Nordens, um Gesetzgebungen auszuarbeiten, die ihren eigenen Entwicklungsinteressen gerecht werden, und um ihre Standpunkte innerhalb der WTO (insbesondere im Hinblick auf die für 1999 vorgesehene Revision des Artikels $27.3 \mathrm{~b}$ und des gesamten TRIPs-Abkommens im Jahr 2000) besser durchzusetzen.

- Den Ländern des Südens dürfen keine patentrechtlichen Regelungen über lebende Organismen aufgezwungen werden. Sie müssen die Möglichkeit besitzen, selber eigenständige Systeme ( « sui generis») oder komplementäre Bestimmungen ( Privilegien» und «Rechte der Bauern») auszuarbeiten.

- Das «Landwirteprivileg», Samen einer geschützten Pflanzensorte bei der nächsten Aussaat zu verwenden, muss ein unveräusserliches Recht bleiben. Gleichzeitig muss die Bedeutung des traditionellen Saatguts hervorgehoben werden.

- Es muss ein Schutz für die informellen Erfindungen der lokalen Gemeinschaften des Südens ausgearbeitet werden. Die von der FAO formulierten «Rechte der Bauern" sowie die Aufgabe des zu diesem Zweck errichteten internationalen Fonds müssen konkretisiert werden. Das Engagement der FAO muss, sobald die Überarbeitung und Präzisierung abgeschlossen sind, zu einem international geltenden Abkommen mit zwingendem Charakter erhoben werden (z.B. als Zusatzprotokoll zum Übereinkommen über die biologische Vielfalt).

\section{LITERATURHINWEISE}

J. Bunders/B. Haverkort/W. Hiemstra, «Biotechnology : Building on Farmers'Knowledge », Macmillan, London, 1996.

C. Correa/WHO, «The Uruguay Round and Drugs », Geneva, 1997.

A. Cosbey/IISD, «The Sustainable Development Effects of the WTO TRIPs Agreement », Winnipeg, 1996.

N. Keim/Communauté de travail Swissaid/Action de carême/Pain pour le prochain/Helvetas/Caritas, «Brevet sur la vie? », Revue Sud 3/octobre 1997, Berne/Lausanne

W. Lesser/UNEP, «Institutional Mechanisms supporting Trade in Genetic Materials », Geneva, 1994.

G. Singh Nijar/TWN, «TRIPs and Biodiversity: A Third World View», Penang, 1996.

Ouvrage collectif/CRDI, «Un brevet pour la vie», Ottawa, 1994.

RAFI/UNDP, «Conserving Indigenous Knowledge: Integrating two Systems of Innovation », New York, 1994.

UICN, «Guide de la Convention sur la diversité biologique», Gland, 1994.

UNCTAD, « The TRIPs Agreement and Developing Countries », Geneva, 1996.

WWF International, «The UN Biodiversity Convention and the WTO TRIPs Agreement », Gland, 1995. 